\title{
NLO QCD corrections to WW+jet production including leptonic W decays at hadron colliders
}

\author{
Stefan Dittmaier ${ }^{a}$, Stefan Kallweit ${ }^{* b}$ and Peter Uwer ${ }^{c} \dagger$ \\ ${ }^{a}$ Albert-Ludwigs-Universität Freiburg, Physikalisches Institut, D-79104 Freiburg, Germany \\ ${ }^{b}$ Paul Scherrer Institut, Würenlingen und Villigen, CH-5232 Villigen PSI, Switzerland \\ ${ }^{c}$ Institut für Physik, Humboldt-Universität zu Berlin, D-10099 Berlin, Germany \\ E-mail: stefan.dittmaierephysik.uni-freiburg.de, \\ stefan.kallweitepsi.ch, Peter.Uwerephysik.hu-berlin.de
}

We report on the calculation of the next-to-leading order QCD corrections to the production of W-boson pairs in association with a hard jet at the Tevatron and the LHC, which is an important source of background for Higgs and new-physics searches. Leptonic decays of the $\mathrm{W}$ bosons are included by applying an improved version of the narrow-width approximation that treats the $\mathrm{W}$ bosons as on-shell particles, but keeps the information on the $\mathrm{W}$ spin. A selection of differential NLO QCD cross sections is provided both for the LHC and the Tevatron. The QCD corrections stabilize the LO prediction for the cross section with respect to scale variations. The differential LO cross sections are generally not simply rescaled by the corrections. Their shapes are particularly distorted if an additional energy scale is involved.

RADCOR 2009 - 9th International Symposium on Radiative Corrections (Applications of Quantum Field Theory to Phenomenology)

October 25-30 2009

Ascona, Switzerland

*Speaker.

$\dagger$ This work is supported in part by the European Community’s Marie-Curie Research Training Network HEPTOOLS under contract MRTN-CT-2006-035505, by the DFG Sonderforschungsbereich/Transregio 9 "Computergestützte Theoretische Teilchenphysik" SFB/TR9, and by the Initiative and Networking Fund of the Helmholtz Association, contract HA-101 ("Physics at the Terascale"). 


\section{Introduction}

The search for new-physics particles-including the Standard Model Higgs boson-will be the primary task in high-energy physics in the era of the LHC. The extremely complicated hadron collider environment does not only require sufficiently precise predictions for new-physics signals, but also for many complicated background reactions that cannot entirely be measured from data. Among such background processes, several involve three, four, or even more particles in the final state, rendering the necessary next-to-leading-order (NLO) calculations in QCD very complicated. This problem lead to the creation of an “experimenters' wishlist for NLO calculations” [1, 2, 的 that were still missing at that time, but are required for successful LHC analyses. The process $\mathrm{pp} \rightarrow \mathrm{W}^{+} \mathrm{W}^{-}+\mathrm{jet}+\mathrm{X}$ made it to the top of this list. Meanwhile the $2 \rightarrow 3$ particle processes and

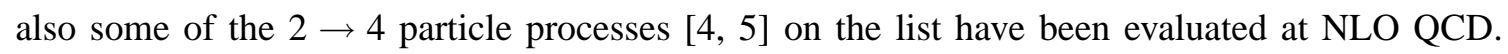
Moreover, benchmark results for the virtual corrections have been presented for a specific phasespace point for all $2 \rightarrow 4$ processes on the list in Ref. [6].

The process of $\mathrm{WW}+\mathrm{jet}$ production is an important source for background to the production of a Higgs boson that subsequently decays into a W-boson pair, where additional jet activity might arise from the production. WW+jet production delivers also potential background to new-physics searches, such as supersymmetric particles, because of leptons and missing transverse momentum from the $\mathrm{W}$ decays. Besides the process is interesting in its own right, since $\mathrm{W}$-pair production processes enable a direct analysis of the non-abelian gauge-boson self-interactions, and a large fraction of W pairs will show up with additional jet activity at the LHC. Last but not least WW+jet at NLO also delivers the real-virtual contributions to the next-to-next-to-leading-order (NNLO) calculation of W-pair production, for which further building blocks are presented in Ref. [7].

Here we report on the calculation of the process $\mathrm{pp} / \mathrm{p} \overline{\mathrm{p}} \rightarrow \mathrm{W}^{+} \mathrm{W}^{-}+\mathrm{jet}+\mathrm{X}$ in NLO QCD including leptonic W-boson decays. Results of this calculation have been published in Refs. [8, 9]. Parallel to our work, another NLO study [10] of $\mathrm{pp} \rightarrow \mathrm{W}^{+} \mathrm{W}^{-}+$jet $+\mathrm{X}$ at the LHC appeared. Moreover, NLO QCD corrections to the related processes $\mathrm{pp} \rightarrow \mathrm{W} \gamma+\mathrm{jet}+\mathrm{X}$ [11] and $\mathrm{pp} \rightarrow \mathrm{ZZ}+\mathrm{jet}+\mathrm{X}$ [12] have been calculated recently.

\section{Details of the NLO calculation}

At leading order (LO), hadronic $\mathrm{WW}+$ jet production receives contributions from the partonic processes $\mathrm{q} \overline{\mathrm{q}} \rightarrow \mathrm{W}^{+} \mathrm{W}^{-} \mathrm{g}$, $\mathrm{qg} \rightarrow \mathrm{W}^{+} \mathrm{W}^{-} \mathrm{q}$, and $\mathrm{g} \overline{\mathrm{q}} \rightarrow \mathrm{W}^{+} \mathrm{W}^{-} \overline{\mathrm{q}}$, where $\mathrm{q}$ stands for up- or downtype quarks. Note that the amplitudes for $\mathrm{q}=\mathrm{u}, \mathrm{d}$ are not the same, even for vanishing light-quark masses. All three channels are related by crossing symmetry.

The leptonic $\mathrm{W}$ decays are implemented by means of an improved narrow-width approximation (NWA) that treats the W bosons as on-shell particles, but keeps the spin correlations between production and decay processes. In this way, a significantly better approximation of the full calculation is achieved, which can be read off the comparison of the sample LO distributions in Figure 1 .

In order to prove the correctness of our results we have evaluated each ingredient twice using independent calculations based—as far as possible—on different methods, yielding results in mutual agreement. 

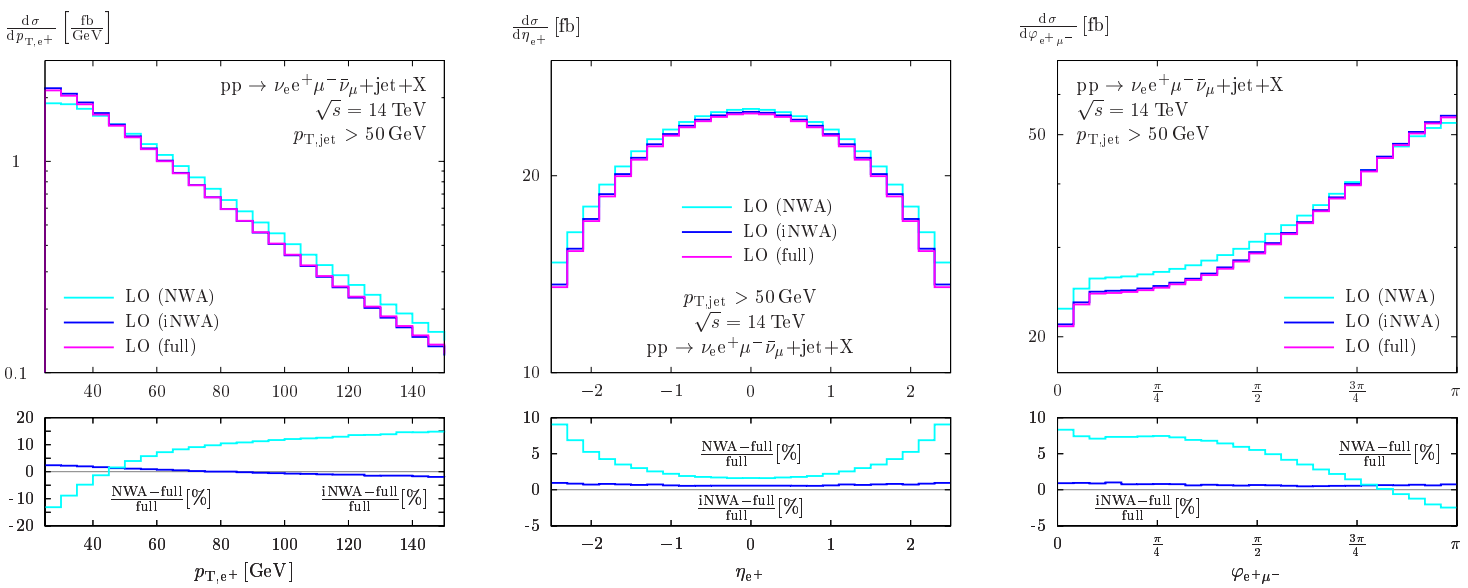

Figure 1: Comparison of W-decay descriptions in the distributions of the transverse momentum of $\mathrm{e}^{+}$(left plot), the pseudo-rapidity of $\mathrm{e}^{+}$(central plot), and the azimuthal angle between the two decay leptons (right plot). The LO cross sections are evaluated at $\mu=\mu_{\mathrm{fact}}=\mu_{\mathrm{ren}}=M_{\mathrm{W}}$ for the full calculation, the naive NWA, and the improved NWA. (Taken from Ref. [9].)

\subsection{Virtual corrections}

Version 1 of the virtual corrections is essentially obtained as for the related processes of $\operatorname{tt} H$ [13] and $\bar{t}+$ jet [14] production. The Feynman diagrams are generated with FeynArts 1.0 [15] and further processed with in-house Mathematica routines, which automatically create an output in Fortran. The IR divergences (soft and collinear) are analytically separated from the finite remainder in terms of triangle subdiagrams, as described in Refs. [13, 16]. This separation, in particular, allows for a transparent evaluation of so-called rational terms that originate from $D$-dependent terms multiplying IR divergences, which appear as single or double poles in $\varepsilon$. As generally shown in Ref. [廿], after properly separating IR from UV divergences such rational terms originating from IR divergences completely cancel; this general result is confirmed in our explicit calculation. For the results presented in Ref. [8], the pentagon tensor integrals were directly reduced to box integrals following Ref. [17], while box and lower-point integrals were reduced à la Passarino-Veltman [18] to scalar integrals. This procedure completely avoids inverse Gram determinants of external momenta in the reduction step from 5-point to 4-point integrals, but the reduction of box and lower-point tensor integrals involves such inverse determinants via the Passarino-Veltman algorithm. Although these inverse determinants jeopardize the numerical stability in regions where such determinants are small, sufficient numerical stability was already achieved. Meanwhile the tensor reduction has been further improved using the methods of Ref. [19]. The scalar one-loop integrals are either calculated analytically or using the results of Refs. [20, 21, 22].

Version 2 of the evaluation of loop diagrams starts with the generation of diagrams and amplitudes via FeynArts 3.4 [23] which are then further manipulated with FormCalc 6.0 [24] and eventually automatically translated into Fortran code. The whole reduction of tensor to scalar integrals is done with the help of the LoopTools library [24], which employs the method of Ref. [17] for the 5-point tensor integrals, Passarino-Veltman [18] reduction for the lower-point tensors, and the FF package [25, 26] for the evaluation of regular scalar integrals. The dimensionally regularized soft or collinear singular 3- and 4-point integrals had to be added to this library. To this end, the 

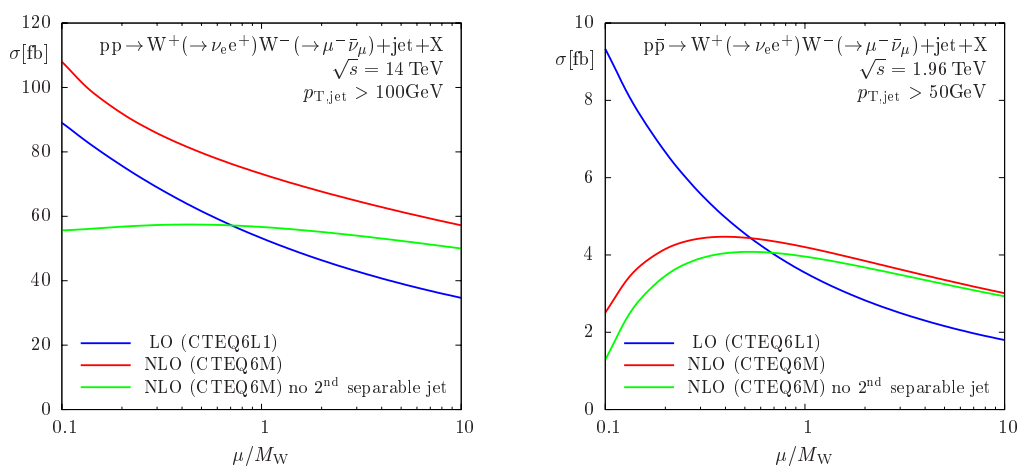

Figure 2: Scale dependence of the WW+jet cross sections with $\mathrm{W}$ decays included and further cuts applied according to Ref. [9]. For the LHC setup, the results are given for $p_{\mathrm{T}, \mathrm{jet}}>100 \mathrm{GeV}$ (left plot). For the Tevatron we show results for $p_{\mathrm{T}, \mathrm{jet}}>50 \mathrm{GeV}$ (right plot).

explicit results of Ref. [16] for the vertex and of Ref. [27] for the box integrals (with appropriate analytical continuations) are taken.

\subsection{Real corrections}

The matrix elements for the real corrections are given by the processes $0 \rightarrow \mathrm{W}^{+} \mathrm{W}^{-} \mathrm{q} \overline{\mathrm{q} g g}$ and $0 \rightarrow \mathrm{W}^{+} \mathrm{W}^{-} \mathrm{q} \overline{\mathrm{q}} \mathrm{q}^{\prime} \overline{\mathrm{q}}^{\prime}$ with a large variety of flavour insertions for the light quarks $\mathrm{q}$ and $\mathrm{q}^{\prime}$. The partonic processes are obtained from these matrix elements by all possible crossings of quarks and gluons into the initial state. The evaluation of the real-emission amplitudes is performed in two independent ways. In one approach we apply the Weyl-van-der-Waerden formalism (as described in Ref. [28]). The other one is based on Madgraph [29] generated code. Both evaluations employ (independent implementations of) the dipole subtraction formalism [30] for the extraction of IR singularities and for their combination with the virtual corrections.

In one calculation the phase-space integration is performed by a multi-channel Monte Carlo integrator [31] with weight optimization [32] written in $\mathrm{C++}$, which is constructed similar to Racoon $W W[33$, 34]. The second calculation uses a simple mapping where the phase space is generated from a sequential splitting.

\section{Numerical results}

We consistently use the CTEQ6 [35] set of parton distribution functions (PDFs), i.e. we take CTEQ6L1 PDFs with a 1-loop running $\alpha_{\mathrm{s}}$ in LO and CTEQ6M PDFs with a 2-loop running $\alpha_{\mathrm{s}}$ in NLO. The complete setup we used for our numerical calculations is precisely defined in Ref. [9], where a large variety of additional results is provided.

Figure 2 shows the scale dependence of the NLO cross section for the LHC and the Tevatron. The QCD corrections stabilize the LO prediction for the WW+jet cross section considerably with respect to a variation of the factorization and renormalization scales which we identify with each other. At the LHC, this stabilization of the prediction, however, requires a veto on a second hard jet. Otherwise the production of final states with WW+2jets, which yields a LO component of the NLO correction, introduces again a large scale dependence. In Figure 3 a sample of NLO distributions is 


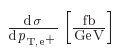
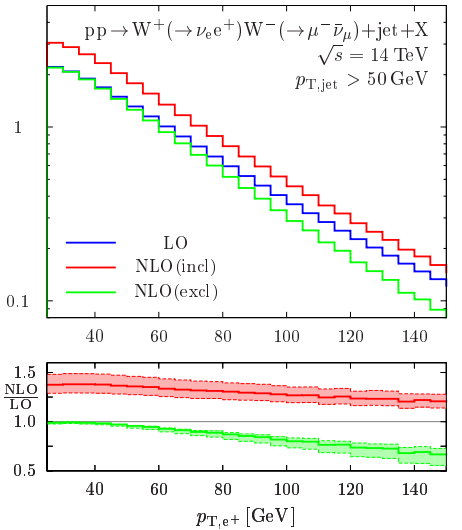

$\frac{\mathrm{d} \sigma}{\mathrm{d} \eta_{\mathrm{e}}+}[\mathrm{fb}]$
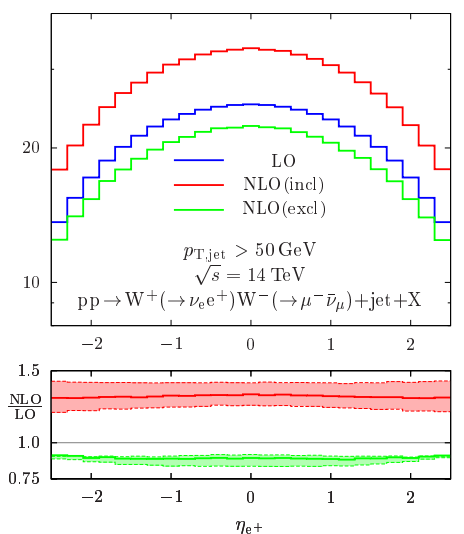
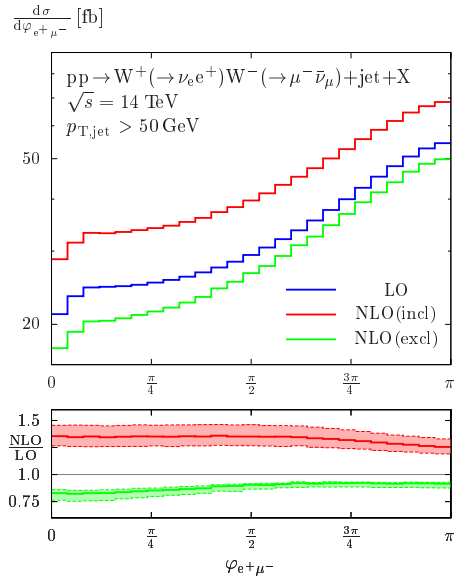

Figure 3: Differential cross sections for WW+jet with decays included in the improved NWA at the LHC: The LO and NLO distributions are shown for $\mu=\mu_{\mathrm{fact}}=\mu_{\mathrm{ren}}=M_{\mathrm{W}}$. The distributions of the transverse momentum of $\mathrm{e}^{+}$(left plot), the pseudo-rapidity of $\mathrm{e}^{+}$(central plot), and the azimuthal angle between the two decay leptons (right plot) are depicted. The bands in the $K$-factors refer to a variation of $\mu$ by a factor of 2 in the NLO quantities. (Taken from Ref. [9].)

provided for the LHC setup. At the LHC the psuedo-rapidity distributions in the dominant region and also the distributions in the angles between the two charged leptons have an almost constant $K$-factor of about 1.3 (inclusive cross-section definition); for the exclusive cross-section definition the corrections are even smaller and rather close to 1 . The $p_{\mathrm{T}}$ spectra, on the other hand, show a much more phase-space-dependent $K$-factor with the exclusive cross-section definition showing an even larger dependence than the inclusive one. This is not surprising since the $p_{\mathrm{T}}$ introduces an additional scale which could introduce potentially large logarithms which are badly treated by a constant renormalization scale. At the Tevatron our findings are similar [8], 9]. We note that the almost constant $K$-factor which holds for a remarkable number of distributions has also been observed in Ref. [10].

\section{References}

[1] C. Buttar et al. [QCD, EW, and Higgs Working Group], arXiv:hep-ph/0604120.

[2] J. M. Campbell, J. W. Huston and W. J. Stirling, Rept. Prog. Phys. 70 (2007) 89 [arXiv:hep-ph/0611148].

[3] Z. Bern et al. [NLO Multileg Working Group], arXiv:0803.0494 [hep-ph].

[4] A. Bredenstein, A. Denner, S. Dittmaier and S. Pozzorini, JHEP 0808 (2008) 108 [arXiv:0807.1248 [hep-ph]] and Phys. Rev. Lett. 103 (2009) 012002 [arXiv:0905.0110 [hep-ph]];

G. Bevilacqua, M. Czakon, C. G. Papadopoulos, R. Pittau and M. Worek, arXiv:0907.4723 [hep-ph].

[5] R. K. Ellis, W. T. Giele, Z. Kunszt, K. Melnikov and G. Zanderighi, JHEP 0901 (2009) 012 [arXiv:0810.2762 [hep-ph]];

R. K. Ellis, K. Melnikov and G. Zanderighi, JHEP 0904 (2009) 077 [arXiv:0901.4101 [hep-ph]] and arXiv:0906.1445 [hep-ph];

C. F. Berger et al., Phys. Rev. Lett. 102 (2009) 222001 [arXiv:0902.2760 [hep-ph]] and arXiv:0907.1984 [hep-ph]. 
[6] A. van Hameren, C. G. Papadopoulos and R. Pittau, arXiv:0903.4665 [hep-ph].

[7] G. Chachamis, M. Czakon and D. Eiras, JHEP 0812 (2008) 003 [arXiv:0802.4028 [hep-ph]] and arXiv:0806.3043 [hep-ph].

[8] S. Dittmaier, S. Kallweit and P. Uwer, Phys. Rev. Lett. 100 (2008) 062003 [arXiv:0710.1577 [hep-ph]].

[9] S. Dittmaier, S. Kallweit and P. Uwer, Nucl. Phys. B 826, 18 (2010) [arXiv:0908.4124 [hep-ph]].

[10] J. M. Campbell, R. Keith Ellis and G. Zanderighi, JHEP 0712 (2007) 056 [arXiv:0710.1832 [hep-ph]].

[11] F. Campanario, C. Englert, M. Spannowsky and D. Zeppenfeld, Europhys. Lett. 88 (2009) 11001 [arXiv:0908.1638 [hep-ph]].

[12] T. Binoth, T. Gleisberg, S. Karg, N. Kauer and G. Sanguinetti, arXiv:0911.3181 [hep-ph].

[13] W. Beenakker, S. Dittmaier, M. Krämer, B. Plümper, M. Spira and P. M. Zerwas, Nucl. Phys. B 653 (2003) 151 [arXiv:hep-ph/0211352].

[14] S. Dittmaier, P. Uwer and S. Weinzierl, Phys. Rev. Lett. 98 (2007) 262002 [arXiv:hep-ph/0703120] and Eur. Phys. J. C 59 (2009) 625 [arXiv:0810.0452 [hep-ph]].

[15] J. Küblbeck, M. Böhm and A. Denner, Comput. Phys. Commun. 60 (1990) 165.

[16] S. Dittmaier, Nucl. Phys. B 675 (2003) 447 [arXiv:hep-ph/0308246].

[17] A. Denner and S. Dittmaier, Nucl. Phys. B 658 (2003) 175 [arXiv:hep-ph/0212259].

[18] G. Passarino and M. J. G. Veltman, Nucl. Phys. B 160 (1979) 151.

[19] A. Denner and S. Dittmaier, Nucl. Phys. B 734, 62 (2006) [arXiv:hep-ph/0509141].

[20] G. 't Hooft and M. J. G. Veltman, Nucl. Phys. B 153, 365 (1979).

[21] W. Beenakker and A. Denner, Nucl. Phys. B 338 (1990) 349.

[22] A. Denner, U. Nierste and R. Scharf, Nucl. Phys. B 367, 637 (1991).

[23] T. Hahn, Comput. Phys. Commun. 140 (2001) 418 [arXiv:hep-ph/0012260].

[24] T. Hahn and M. Perez-Victoria, Comput. Phys. Commun. 118 (1999) 153 [arXiv:hep-ph/9807565].

[25] G. J. van Oldenborgh and J. A. M. Vermaseren, Z. Phys. C 46, 425 (1990).

[26] G. J. van Oldenborgh, Comput. Phys. Commun. 66 (1991)

[27] Z. Bern, L. J. Dixon and D. A. Kosower, Nucl. Phys. B 412 (1994) 751 [arXiv:hep-ph/9306240].

[28] S. Dittmaier, Phys. Rev. D 59 (1999) 016007 [arXiv:hep-ph/9805445].

[29] T. Stelzer and W. F. Long, Comput. Phys. Commun. 81 (1994) 357 [arXiv:hep-ph/9401258].

[30] S. Catani and M. H. Seymour, Nucl. Phys. B 485 (1997) 291 [Erratum-ibid. B 510 (1998) 503] [arXiv:hep-ph/9605323].

[31] F. A. Berends, R. Pittau and R. Kleiss, Nucl. Phys. B 424 (1994) 308 [arXiv:hep-ph/9404313].

[32] R. Kleiss and R. Pittau, Comput. Phys. Commun. 83, 141 (1994) [arXiv:hep-ph/9405257].

[33] A. Denner, S. Dittmaier, M. Roth and D. Wackeroth, Nucl. Phys. B 560 (1999) 33 [arXiv:hep-ph/9904472].

[34] M. Roth, doctoral thesis, DISS-ETH-13363, 1999, arXiv:hep-ph/0008033.

[35] J. Pumplin et al., JHEP 0207 (2002) 012 [arXiv:hep-ph/0201195]. 